\title{
Modeling of Ionization in a Spherical Surface Ionizer
}

\author{
M. TUREK* \\ Institute of Physics, Maria Curie-Skłodowska University in Lublin \\ pl. M. Curie-Skłodowskiej 1, 20-031 Lublin, Poland
}

\begin{abstract}
A Monte Carlo method-based model of a hot cavity surface ion source with a spherically shaped ionizer is presented. A numerical code enables studies of ion source efficiency as a function of an extraction voltage, geometry of the ionizer and its temperature, the size of extraction aperture and many other factors. The calculation results for a variety of mentioned parameters are presented. A novel configuration of surface ion source is proposed and discussed - the efficiency of the source with an almost spherically shaped ionizer could be much higher than that of currently used constructions of ion sources.
\end{abstract}

PACS: 07.77.Ka, 07.05.Tp, 34.35.+a

\section{Introduction}

Particular needs of various applications in the fields of nuclear spectroscopy, material science, micro- and optoelectronics, isotope separation, medicine and many others resulted in a multitude of ion source constructions [1]. One of them is a surface ionization source equipped with a hot cavity ionizer characterized by small dimensions and a simple design. Despite the fact it was invented many years ago $[2,3]$ it is still used and developed $[4,5]$, mostly due to its numerous advantages such as: purity of beam, low energy spread of extracted beam, short time ions stay in the ionizer, which could be a clue in the case of short-lived isotopes investigations. The other important advantages of hot cavity ion sources are: their high ionization efficiency and a small amount of substance needed to obtain stable ion current as well as low energy spread of the obtained beam.

The paper focuses on modeling of a source with a spherical ionizer, one of numerous variations of surface ion sources. Sources of that type were successfully used both for negative and positive ion beam production $[6,7]$.

There are numerous theoretical approaches describing surface ionization sources as well as the effect of ionization efficiency amplification by a hot cavity [8-10], usually a tubular ionizer is considered. It was shown that the formula obtained for a spherical cavity [11] describes well simulation results obtained for a tubular ionizer [12]. However, only a few attempts have been made to describe processes (as vapor transport from an irradiated target to the ionizer [13] or release curves dependence on the ionizer's geometry [14]) in the hot cavity surface ionization sources by means of computer simulations.

The aim of this paper is to present a Monte Carlo method based on a numerical model enabling simula-

\footnotetext{
* e-mail: mturek@kft.umcs.lublin.pl
}

tions of processes inside the spherical ionizer. The paper presents the dependences of source ionization efficiency on different working parameters like extraction voltage, diameter of extraction opening, distance between the source and extraction electrode and the like. An example of the source's efficiency dependence on ionizer temperature is also shown. It will be shown that the presented code is able to support ion source design and optimization. Two different configurations of sources are considered. One of them resembles much that used in existing sources with hemispherical ionizer, whilst the novel one, having a kind of cavity, is characterized by much larger ionization efficiency.

\section{Surface ionization ion source}

The main part of a classic hot cavity surface ionization source is an elongated, semi-opened tube made of refractory metal known as an ionizer. It is heated to high working temperature $T(\approx 2500 \mathrm{~K})$ by beams of electrons or by ohmic heating. Atoms of the substance introduced into the ionizer collide with hot walls and are ionized with a probability given by the Saha-Langmuir formula [15]. According to it the rate of ions and atoms, known as ionization degree, is written as

$$
\alpha=G \exp \left(-\left(V_{\mathrm{i}}-\phi_{\mathrm{e}}\right) / k T\right),
$$

where $V_{\mathrm{i}}$ and $\phi_{\mathrm{e}}$ are the ionization potential of an atom and the work function of the ionizer material, respectively, and $G$ is the constant characteristics of a given kind of atom. The formula (1) refers to positive ion production. In the case of negative ions $V_{\mathrm{i}}-\phi_{\mathrm{e}}$ has to be substituted by $\phi_{\mathrm{e}}-E_{\mathrm{a}}$, where $E_{\mathrm{a}}$ is the electron affinity of an atom. Another frequently used quantity describing ionization is an ionization coefficient $\beta=\alpha /(1+\alpha)$, which could be understood as the probability of ionization during a collision. The above-defined quantities describe a single act of ionization, and should not be mixed up with 
a total source ionization efficiency, a quantity that is defined as a ratio of the number of ions $N_{+}$and the number of all particles passing the extraction opening

$$
\beta_{\mathrm{s}}=N_{+} /\left(N_{+}+N_{0}\right),
$$

where $N_{0}$ is the number of neutral particles.

The efficiency of the source could be very high due to the fact that particles undergo multiple collisions with the ionizer surface. Hence, the total probability of ionization could be much higher than that predicted by (1).

Over many years surface ionization ion sources were used for positive ion production: there were several high-work function metals suitable for ionizers. On the contrary, there was the lack of stable low-work function materials for negative ion source ionizers. Lanthanum hexaboride has become the most popular material $[16,17]$, mostly due to the very low work function: $2.3-3.2 \mathrm{eV}$ [7]. Spherically shaped ionizers were used to enhance ionization and to focus the ion beam. Spherical ionizers are also widely used in sputtering ion sources [18] for production of primary ion beams (e.g. $\left.\mathrm{Cs}^{+}\right)$that are accelerated towards a sputtered electrode.

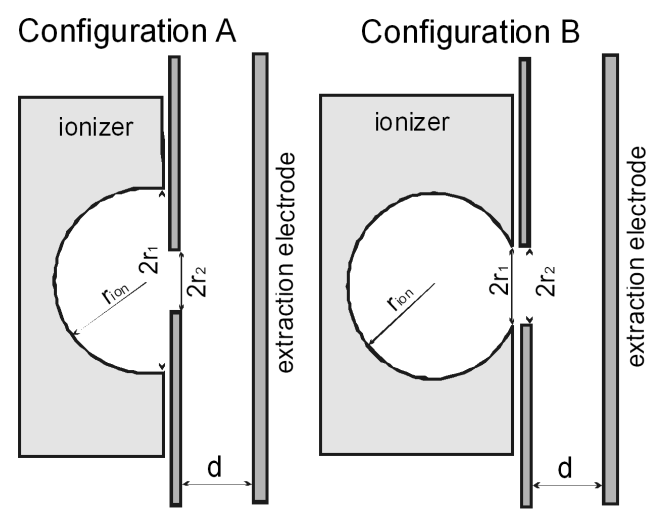

Fig. 1. Schematic views of the surface ion sources: geometry of existing ion sources (A) and novel configuration (B) postulated in the paper.

Two different ion source configurations, shown schematically in Fig. 1, are under investigation. The first of them (A) resembles that employed in experiments for both negative and positive ion production $[6,7,16,17]$. In that case, a hemispherical ionizer closed by a thin plate with the extraction hole is used. Another configuration we propose (B) is a spherical cavity with small extraction opening. It will be shown that such shape of the ionizer leads to source efficiency higher than in the case A, due to a stronger effect of multiple collisions with walls.

\section{Numerical model}

The numerical code is a kind of test particle code solving equations of motion for particles in the cavity. A flat extraction electrode is considered for simplicity reasons. The electrode is on the potential $-U_{\text {ext }}$ with respect to the ionizer. The distance $d$ between the electrode and the extraction opening is varied within a range of a few millimeters. The electrostatic field penetrates the ionizer at some depth and changes trajectories of ions. The simulation area is covered by a 3D Cartesian spatial grid with the cell dimensions $\Delta x=\Delta y=\Delta z=5 \times 10^{-6} \mathrm{~m}$. The electrostatic potential is found by solving Laplace equation (via successive over-relaxation technique [19]), taking into account boundary conditions like electrode potentials etc.

The model assumes that particles are emitted from the internal surface of the ionizer at random directions. Initial velocities correspond to the ionizer temperature $T$. Particle collisions are neglected, hence, the increase of $\beta_{\mathrm{s}}$ values is caused mainly by multiple collisions of particles with walls. Equations of motion are numerically solved using the 4th order Runge-Kutta method. Electrostatic field at particle's positions is derived by linear interpolation using values at the neighboring nodes. When the particle hits the wall, a Monte Carlo based subroutine decides whether the particle is ionized/neutralized or not, according to the probability $\beta$. A new initial velocity is calculated and the particle keeps on moving until it is caught by extraction field and passes the extraction aperture. The code counts ions and neutral atoms leaving the ionizer and calculates according to (2).

\section{Simulation results}

This section presents the results of simulations performed for two different configurations of the ion source, shown in Fig. 1. The configuration A resembles that employed in manufactured ion sources. A hemispherical ionizer of inner radius $r_{\text {ion }}$ is closed by an end cap with an extraction aperture of radius $r_{2}$. A flat extraction electrode is at the distance $d$ from the source. The main feature of the configuration $\mathrm{B}$ is more of a concave shape of the ionizer $\left(r_{1}=r_{2}<r_{\text {ion }}\right)$. As a result of such concave ionizer's shape a particle undergoes many collisions with its walls. Consequently, this should lead to significant enhancement of $\beta_{\mathrm{s}}$.

Calculations of the influence of extraction voltage $U_{\text {ext }}$ on $\beta_{\mathrm{s}}$ were done for the two geometries. In both cases $r_{\text {ion }}=2 \mathrm{~mm}$ and $r_{2}=0.5 \mathrm{~mm}$ were chosen. Additionally, in configuration A, $r_{1}$ is equal to $r_{\text {ion. Simulations were }}$ done using 50000 of test particles. The mass of a particle was set to 150 a.m.u. The time step of simulation was $5 \times 10^{-9} \mathrm{~s}$. The extraction electrode was placed at the distance of $d=2 \mathrm{~mm}$. The simulation results are shown in Fig. 2a and b. First, the model was validated by checking that for $U_{\text {ext }}=0$ the code provides the theoretically predicted curve $\beta_{\mathrm{s}}(\beta)=\beta$ (not presented). Then the value of $U_{\text {ext }}$ was increased up to $0.5 \mathrm{kV}$ (A) and $2 \mathrm{kV}$ (B) shifting the $\beta_{\mathrm{s}}(\beta)$ curves upwards. In the first case the increase of $U_{\text {ext }}$ for the substances of small ionization coefficient $(\beta=0.01)$ enhances $\beta_{\mathrm{s}}$ triple compared to the Saha-Langmuir estimations. But the efficiency enhancement in the case $B$ is much more visible. The same value of $U_{\text {ext }}=0.5 \mathrm{kV}$ raises $\beta_{\mathrm{s}} 15$ times. The increase of $U_{\text {ext }}$ 
above $0.5 \mathrm{kV}$ seems to be pointless, $\beta_{\mathrm{s}}$ does not increase much. It is worth noticing that the saturation of efficiency enhancement in the case B is observed for larger voltage $(\approx 2 \mathrm{kV})$. As a result, $\beta_{\mathrm{s}}$ is amplified by a factor of $30(\beta=0.01)$ or even more.
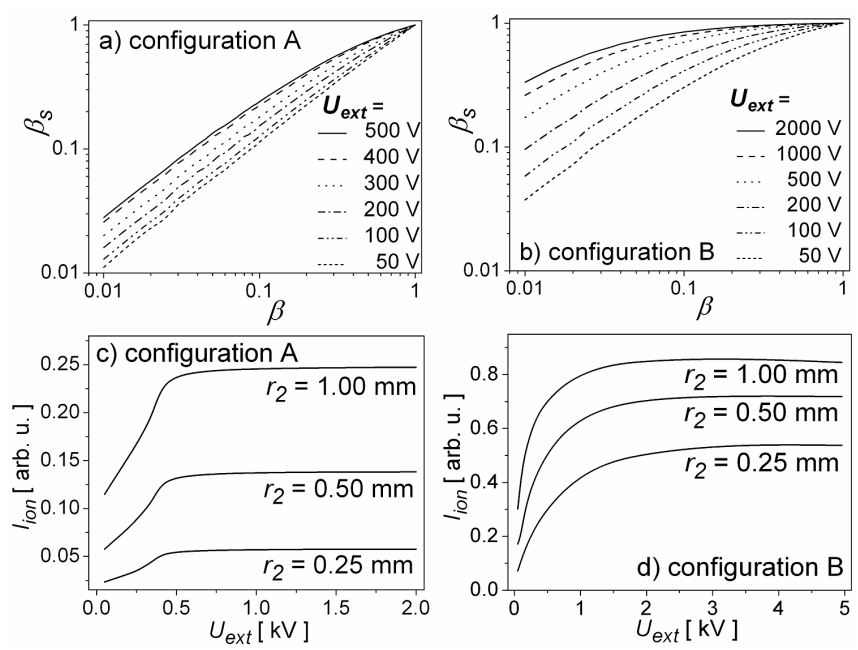

Fig. 2. Dependences of total ion source ionization efficiency on $\beta$ for different values of $U_{\text {ext }}(\mathrm{a}, \mathrm{b})$ and currentvoltage curves for different values of $r_{2}(\mathrm{c}, \mathrm{d})$.

Figures 2c and d show the calculated current-voltage characteristics for both configurations. In the case A one can see an initially fast growth of the current, followed by saturation for $U_{\text {ext }}>0.5 \mathrm{kV}$, which happens when the field is strong enough to catch and extract all ions immediately after their creation. The shape of curves resembles experimental characteristics in [7]. In the case $\mathrm{B}$ the transition to the saturation phase is not as rapid as previously and occurs for larger $U_{\text {ext }}$. The shape of the curves is similar to the trends of experimental data obtained for a tubular ionizer [20].

The influence of the size of extraction aperture $r_{2}$ on $\beta_{\mathrm{s}}$ was also under investigation (Fig. $3 \mathrm{a}$ and b). Calculations were done for $r_{2}$ changing from $0.5 \mathrm{~mm}$ up to $1.25 \mathrm{~mm}$. In both cases $U_{\text {ext }}=0.5 \mathrm{kV}$ was set, the rest of simulation parameters is the same as previously. In the case A, $\beta_{\mathrm{s}}$ does not depend on $r_{2}$ in the considered range. The behavior of $\beta_{\mathrm{s}}\left(r_{2}\right)$ curves is quite different in the case $\mathrm{B}$. One can see a maximum of $\beta_{\mathrm{s}}\left(r_{2}\right)$ curve for $r_{2}=0.65 \mathrm{~mm}$. Increase of $r_{2}$ from its optimal value degrades $\beta_{\mathrm{s}}$ by $25 \%$ (for $1.2 \mathrm{~mm}$ ). In the first case the cavity is shallow and the depth of electric field penetration is sufficient for all ions to be extracted.

Figures $3 \mathrm{c}$ and $\mathrm{d}$ present the dependence of $\beta_{\mathrm{s}}$ on the distance $d$ between the electrode and the extraction hole. In both considered cases $\beta_{\mathrm{s}}$ grows, as the electrode gets closer to the source. Discrepancies of $\beta_{\mathrm{s}}(d)$ trends are caused by different geometry of an ionizer and an end cap. In the case $\mathrm{B}$, the reduction of $d$ from $3 \mathrm{~mm}$ to $0.5 \mathrm{~mm}$ leads to the increase of efficiency by approximately $50 \%$. One may conclude that $d$ should be as small as possible,
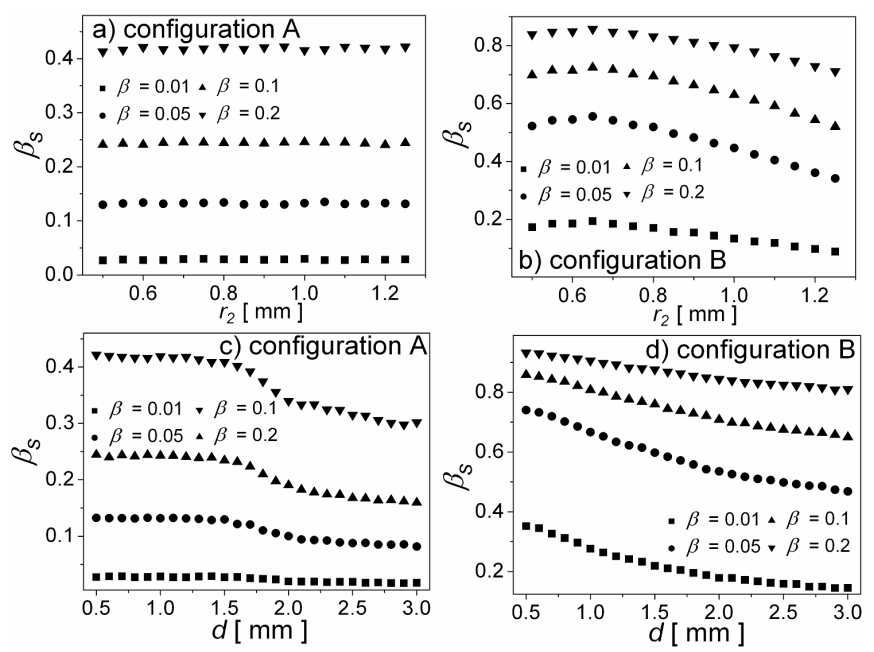

Fig. 3. Dependences of total ion source ionization efficiency on $r_{2}$ for both configurations $(\mathrm{a}, \mathrm{b})$ and influence of distance between the electrode and the ionizer on $\beta_{\mathrm{s}}$ $(\mathrm{c}, \mathrm{d})$.

but in a real source the distance is limited by electric breakdown possibility. The behavior of $\beta_{\mathrm{s}}(d)$ curve is slightly different in the case $\mathrm{A}$. The efficiency also rises with decreasing $d$ but up to some value only $(\approx d=$ $1.5 \mathrm{~mm}$ ). The decrease of $d$ below this value is pointless - the strong extraction field removes all ions from the cavity.

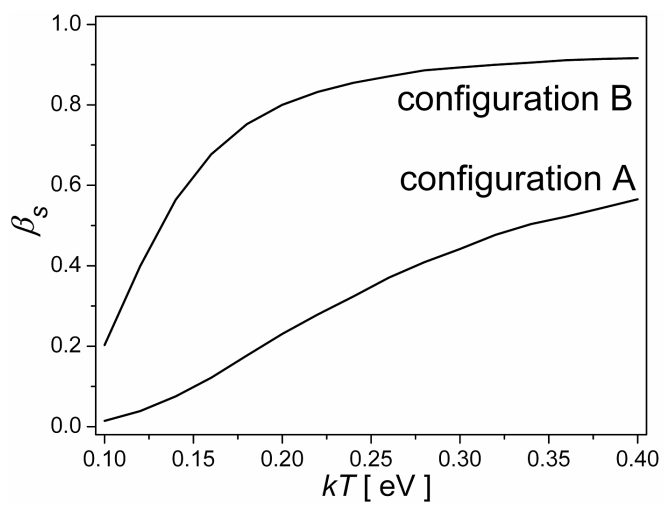

Fig. 4. Total ion source ionization efficiency as a function of ionizer's temperature $T$ for sodium in a tungsten cavity.

The numerical code could also predict influence of temperature $T$ on the total ion source efficiency. Figure 4 shows the results obtained for sodium in a tungsten ionizer. The $U_{\text {ext }}$ was set to $0.5 \mathrm{kV}$ and the electrode was at the distance of $d=2$. Once again a vantage of the new configuration (B) is clear. One should keep in mind that the increase of $\beta_{\mathrm{s}}$ with $T$ is not a general rule. The behavior of $\beta_{\mathrm{s}}(T)$ curves depends on $V_{\mathrm{i}}$ and $\phi_{\mathrm{e}}$ values, as could be deduced from Eq. (1). 


\section{Conclusions}

The paper presents the numerical code for simulations of ionization in a hot cavity surface ion source. The numerical model takes into account the factors like the size and geometry of the ionizer and the extraction system, extraction voltage, ionizer's temperature and the like.

The code provides meaningful information: e.g. current-voltage curves, dependences of $\beta_{\mathrm{s}}$ on the diameter of extraction aperture or the distance from the extraction electrode. Some results (e.g. current-voltage characteristics) are in a good qualitative agreement with the experimental data. Two different geometries of the ionizer were studied. It has been shown that this of the ion source with the almost closed ionizer could be significantly larger than the efficiency of the source with a hemispherical ionizer, which is a popular solution in the currently used surface ionization ion sources. This shows that the code could be an effective and reliable tool supporting the research and design of surface ionization ion sources.

\section{Acknowledgments}

This work is supported by Maria Curie-Skłodowska University in Lublin in the frame of the grant of the MCSU Rector from the Polish Ministry of Science and Higher Education funds.

\section{References}

[1] Physics and Technology of Ion Sources, Ed. I.G. Brown, Wiley-VCH, Weinheim 2004, p. 107.

[2] G.J. Beyer, E. Herrmann, A. Piotrowski, V.I. Raiko, H. Tyroff, Nucl. Instrum. Methods 96, 347 (1971).

[3] A. Latuszyński, K. Zuber, J. Zuber, A. Potempa, W. Żuk, Nucl. Instrum. Methods 120, 321 (1974).
[4] G.D. Alton, Y. Liu, D.W. Stracener, Rev. Sci. Instrum. 77, 03A711 (2006).

[5] A. Osa, S. Ichikawa, M. Matsuda, T.K. Sato, S.-C. Jeong, Nucl. Instrum. Methods Phys. Res. B 266, 4394 (2008).

[6] G.D. Alton, Y. Liu, S.N. Murray, G.D. Mills, H. Zaim, Rev. Sci. Instrum. 73, 796 (2003).

[7] G.D. Alton, Y. Liu, H. Zaim, S.N. Murray, Nucl. Instrum. Methods Phys. Res. B 211, 425 (2003).

[8] A. Latuszyński, V.I. Raiko, Nucl. Instrum. Methods 125, 61 (1975).

[9] R. Kirchner, Nucl. Instrum. Methods 186, 275 (1981).

[10] R. Kirchner, Nucl. Instrum. Methods Phys. Res. A 292, 203 (1990).

[11] A. Latuszyński, K. Pyszniak, A. Droździel, M. Turek, D. Mączka, J. Meldizon, Vacuum 81, 1150 (2007).

[12] M. Turek, K. Pyszniak, A. Drozdziel, Vacuum 83, S260 (2009).

[13] Y. Zhang, G.D. Alton, Nucl. Instrum. Methods Phys. Res. B 241, 947 (2005).

[14] B. Mustapha, J.A. Nolen, Nucl. Instrum. Methods Phys. Res. A 521, 59 (2004).

[15] M.N. Saha, Philos. Mag. 40, 472 (1920).

[16] G.D. Alton, M.T. Johnson, G.D. Mills, Nucl. Instrum. Methods Phys. Res. A 328, 154 (1993).

[17] M. Menna, R. Catherall, J. Lettry, E. Noah, T. Stora, the ISOLDE collaboration, Nucl. Instrum. Methods Phys. Res. B 266, 4391 (2008).

[18] J.R. Southon, M.L. Roberts, Nucl. Instrum. Methods Phys. Res. B 172, 257 (2000).

[19] R. Hockney, J. Eastwood, Computer Simulation Using Particles, Mir, Moscow 1987, p. 212.

[20] A. Latuszyński, D. Mączka, Vacuum 51, 109 (1998). 